\title{
An experiment to study and control the Langmuir sheath around INTERBALL-2
}

\author{
K. Torkar ${ }^{1}$, M. V. Veselov ${ }^{2}$, V. V. Afonin ${ }^{2}$, H. Arends ${ }^{3}$, M. Fehringer ${ }^{4}$, G. Fremuth ${ }^{1}$, K. Fritzenwallner ${ }^{1}$, \\ Y. I. Galperin ${ }^{2}$, A. I. Kozlov ${ }^{5}$, A. Pedersen ${ }^{3}, 6$, S. Perraut ${ }^{7}$, W. Riedler ${ }^{1}$, F. Rüdenauer ${ }^{4}$, R. Schmidt ${ }^{3}$, A. Smit ${ }^{3}$, \\ N. Valavanoglou ${ }^{8}$, L. V. Zinin ${ }^{9}$ \\ ${ }^{1}$ Space Research Institute, Austrian Academy of Sciences, Inffeldgasse 12, A-8010 Graz, Austria \\ E-mail: torkar@fiwf01.tu-graz.ac.at \\ ${ }^{2}$ Space Research Institute, Russian Academy of Sciences, Moscow, Russia \\ ${ }^{3}$ Space Science Division, ESA/ESTEC, NL-2200 AG Noordwijk, The Netherlands \\ ${ }^{4}$ Austrian Research Centre Seibersdorf, A-2444 Seibersdorf, Austria \\ ${ }^{5}$ NPO KVANT, Moscow, Russia \\ ${ }^{6}$ Space Science Division, ESA/ESTEC, NL-2200 AG Noordwijk, The Netherlands \\ ${ }^{7}$ CETP/IPSL, Orleans, France \\ ${ }^{8}$ Department of Communications and Wave Propagation, Technical University Graz, Graz, Austria \\ ${ }^{9}$ Kaliningrad State University, Kaliningrad, Russia
}

Received: 25 August 1997 / revised: 6 May 1998 / Accepted: 11 June 1998.

\begin{abstract}
The satellite INTERBALL-2 has an orbit with high inclination $\left(62.8^{\circ}\right)$, covering the altitude range between a few hundred and about $20000 \mathrm{~km}$. The ambient plasma conditions along this orbit are highly variable, and the interactions of this plasma with the spacecraft body as well as the photo-electron sheath around it are considered to be interesting topics for detailed studies. The electric potential of the spacecraft with respect to the ambient plasma that develops as a result of the current equilibrium reacts sensitively to variations of the boundary conditions. The measurement and eventual control of this potential is a prerequisite for accurate measurements of the thermal plasma. We describe the purpose and technical implementation of an ion emitter instrument on-board INTERBALL-2 utilising ion beams at energies of several thousand electron volts in order to reduce and stabilise the positive spacecraft potential. First results of the active ion beam experiments, and other measures taken on INTERBALL-2 to reduce charging are presented. Furthermore, the approach and initial steps of modelling efforts of the sheath in the vicinity of the INTERBALL-2 spacecraft are described together with some estimates on the resulting spacecraft potential, and effects on thermal ion measurements. It is concluded that even moderate spacecraft potentials as are commonly observed on-board INTERBALL-2 can significantly distort the measurements of ion distribution functions, especially in the presence of strongly anisotropic distributions.
\end{abstract}

\footnotetext{
*Present address: Department of Physics, University of Oslo, Oslo, Norway

Correspondence to: $\mathrm{K}$. Torkar
}

Key words. Space plasma physics (active perturbation experiments; spacecraft sheaths, wakes, charging; instruments and techniques).

\section{Introduction}

The determination of the electric potential of a spacecraft with respect to the ambient plasma and the threedimensional distribution of the potential around the spacecraft, its influence on the on-board plasma measurements, and the eventual control of the potential are long-standing problems in experimental space plasma physics. The problems arise again for each new highaltitude satellite for magnetospheric research due to differences in the shape of the spacecraft, the plasma detectors' fields of view, and ambient plasma characteristics. The problem of disturbed particle distribution functions at low energy nearly always appears for a sunlit satellite outside the plasmasphere. The resulting measurements are, at best, distorted or have gaps for species being repelled by the potential well. Recent progress in the construction of actively controlled ion sources, for example that operating on the GEOTAIL spacecraft over several years (Schmidt et al., 1995) has opened a new endeavour in approaching this old problem. An ion beam emitter instrument called RON AC-004 with similar capabilities was installed on the satellite INTERBALL-2. A brief description of this instrument and its in-flight operations is given in Sect. 3. The generated current modifies the equilibrium conditions and decreases the positive spacecraft potential, thus extending downwards the energy range of reliable thermal plasma measurements. 
While early failures of the high-voltage generators prevented the fulfilment of the detailed measurement program and specific experiments planned with ion beam modulation, some interesting measurements spanning more than three months of operations in various plasma environments were obtained and are being analysed. The interest in these controlled ion beam injections, the related plasma measurements and the models of the three-dimensional electric field distribution around the spacecraft is justified because of their importance for the interpretation of the on-board thermal plasma measurements, but it is also due to the fact that for the first time such measurements have been made on-board a high-altitude spacecraft with very large areas both sunlit and non-sunlit in a period of very low solar activity. It can be assumed that low fluxes of solar X-rays and EUV may result in a reduced production of high-energy photo-electrons at the surface of the spacecraft (mostly from the solar panels coated with indium oxide). Significant variations of the photoemission related to solar rotation and solar cycle (nearly a factor of two) have been derived from the Pioneer Venus Langmuir probe by Brace et al. (1988). Energetic photo-electrons (5 to $15 \mathrm{eV}$ ) generated by soft X-rays and EUV radiation have been identified to contribute to photo-emission in space by Laakso and Pedersen (1994) and by Pedersen (1995) in a study of electric field probe data from several satellites. The known dependence of this radiation on solar activity is consistent with the observed temporal variability of the photo-emission. Thus, large positive spacecraft potentials should be rare under the conditions on INTERBALL-2, which is in qualitative agreement with the observations.

At the same time the Debye length varies greatly along the orbit of the satellite, from tens of centimetres or less to tens of metres or more. For the latter, large values to a first approximation of a model assuming infinite Debye length and neglecting the magnetic field may be applicable. However, such a simple model is of questionable value for Debye lengths of the order of the dimensions of the spacecraft (i.e. from about $1 \mathrm{~m}$ to several metres), if the extending spacecraft structures such as solar panels, antennas, and booms are taken into account. We shall consider some results of the modelling of the three-dimensional electric fields (for infinite Debye length), and of their effects on the measurements of the thermal ion distribution functions. It will be shown that, for strongly anisotropic ion distribution functions, the resulting distortions can prevent a reliable restitution of the original thermal ion distribution function, and thus may cause incorrect interpretation of the data.

Three independent instruments on-board INTERBALL-2, namely IESP-2M, KM-7 and ALFA-3, provide data from which spacecraft potential can be derived. They have different locations on the booms of the spacecraft and, hence, different positions within or outside the sheath around the spacecraft body. The methods of evaluating the spacecraft potential differ as well. Considerable work lies ahead to analyse and compare these data and suitably adjust the three- dimensional electric field models. We hope this effort will support the interpretation of the thermal plasma data. Here, the approaches used and the first results of this work are presented.

\section{Preparations before launch}

\subsection{Measures to ensure uniform electric potential of the outer spacecraft surfaces including the solar panels}

Considering that one of the main goals of the INTERBALL-2 mission consists of the investigation of thermal plasma, appropriate measures have been taken to achieve optimum performance in this area.

It is obvious that an equipotential surface produces a minimum of disturbances in the ambient plasma. The floating potential adjusts itself to the average conditions along the surface, and the electric field near the surface has a perpendicular component only. Any electrically isolated patches on the surface may charge to different electric potentials under the bombardment by energetic particles. Negative potentials of several $\mathrm{keV}$ have been observed at geostationary orbit, which often results in potentially dangerous electric discharges. As a secondary effect, representative modelling of the potential becomes virtually impossible. To prevent such a case, all external satellite surfaces, antennas, and booms were covered by conductive material. Metallised mylar or conductive paint was employed for all surfaces except the solar panels, which were subject to special attention. The solar cells were coated by indium oxide at the front side. This technique has been applied successfully on many spacecraft in the past two decades. The panels were also coated by metallised mylar on the back. As a result, the electrical resistance between any two points of the spacecraft surface did not exceed $1 \mathrm{Ohm}$.

The satellite INTERBALL-2 in flight configuration (Fig. 1) has a very complicated structure. This fact complicates the modelling of the DC electric field distribution around the satellite, even with equipotential surfaces.

\subsection{AC stray electric and magnetic fields \\ from the solar panels: results of electromagnetic compatibility measurements at CNES, Toulouse}

The solar panels used for the spacecraft were not only conductively coated, but were also specially designed to minimise the electric and magnetic fields emerging from the current carrying wiring to the solar cells. These measures minimised the level of disturbances in the ambient plasma introduced by the satellite. The resulting radiated $\mathrm{AC}$ electric and magnetic field levels were measured in the EMC chamber in CNES, Toulouse. The electric stray field measured between 20 and $1100 \mathrm{kHz}$, measured at $1 \mathrm{~m}$ distance, remained below the required value of $\approx 50 \mathrm{~dB} \mu \mathrm{V} / \mathrm{m}$ by 3 to $20 \mathrm{~dB}$, but notably the magnetic stray fields in the frequency range between 50 


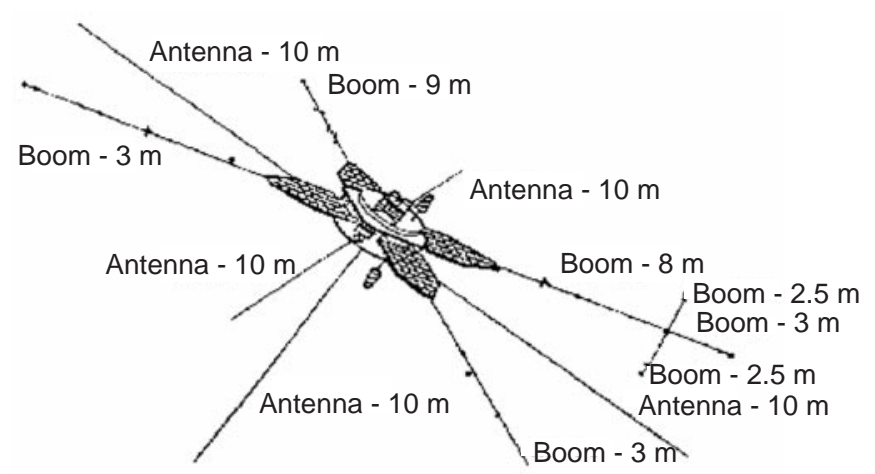

Fig. 1. Structure of the spacecraft INTERBALL-2

and $250 \mathrm{kHz}$, measured at the same distance, were about $60 \mathrm{~dB}$ below the requirements of $60 \mathrm{dBnT}$.

\subsection{Static three-dimensional model of the DC electric field around INTERBALL-2 for infinite Debye length}

As well as pre-flight hardware preparations, some software development had been undertaken, aiming at a better understanding of particle trajectories in the electric field around the spacecraft. Near apogee, when the satellite moves at low velocity in the high-altitude magnetosphere where the plasma density is relatively low, the infinite Debye length approximation for a static field seems to be justifiable. Thus, let us consider such a three-dimensional model of the DC electric field around the INTERBALL-2 satellite. The mathematical model I2 $\mathrm{S}$ of the surface of the INTERBALL-2 (Auroral Probe) satellite includes the following structures:

a. The satellite body, approximated by an equivalent cylinder

b. The solar panels in realistic dimensions

c. All booms for electric and magnetic sensors

d. Five antennas, $10 \mathrm{~m}$ long each, of the POLRAD experiment

The inner boundary condition was a given spacecraft potential $V_{\text {sat }}$, the outer boundary condition was zero potential at infinity (in practice, at distances from the spacecraft larger than about $30 \mathrm{~m}$ ). With these boundary conditions the multigrid solution of the threedimensional Laplace equation for the three-dimensional potential $\varphi$

$\frac{\partial^{2} \varphi}{\partial x^{2}}+\frac{\partial^{2} \varphi}{\partial y^{2}}+\frac{\partial^{2} \varphi}{\partial z^{2}}=0$

was found. For infinite Debye length the shape of the potential distribution can be scaled to any value of $V_{\text {sat }}$, because the electric field is self-similar in units of $V_{\text {sat }}$, as seen from the Laplace equation. The details of the model are described in Zinin et al. (1995).

For the real plasma conditions near the apogee of INTERBALL-2 (at 15000-20000 km altitude), the plas- ma density $N$ usually ranges between 1 and $30 \mathrm{~cm}^{-3}$ and the electron temperature varies, in accordance with $N$, between 3000 and $10000 \mathrm{~K}$. For these conditions the Debye length $D$ becomes:

$$
\begin{aligned}
& D \approx 0.07 \sqrt{\frac{T, \mathrm{~K}}{N, \mathrm{~cm}^{-3}}} \\
& \approx\left\{\begin{array}{lll}
0.7 \mathrm{~m} & & N=30 \mathrm{~cm}^{-3}, T_{e}=3000 \mathrm{~K} \\
& \text { for } & \\
7 \mathrm{~m} & & N=1 \mathrm{~cm}^{-3}, T_{e}=10000 \mathrm{~K} .
\end{array}\right.
\end{aligned}
$$

Although the density might occasionally even be as low as $0.1 \mathrm{~cm}^{-3}$ it is obvious that an infinite Debye length is not a good approximation of the typical real situation, but this assumption is suitable for first order calculations of ion trajectories near the surface (Section 5).

Some examples of the calculations of the threedimensional electric field distribution around INTERBALL-2 are shown in Fig. 2: (1) in the plane of the solar panels (Fig. 2a); and (2) in the plane of "the spacecraft meridian" through the electric field booms (Fig. 2b).

This three-dimensional electric field model was used for calculations of the ion trajectories in the vicinity of the spacecraft entering the on-board plasma instruments for a set of values for $V_{\text {sat }}$ in Sect. 6. Computational difficulties in this straight-forward approach due to the complicated inner boundary condition, the spacecraft surface including solar panels, antennas and booms, are solved by specially designed multigrid algorithms. However, for finite Debye lengths the situation is much more difficult because the structure of the Langmuir sheath around such a body with long extended elements is not well understood. In reality, the supersonic motion of the spacecraft is known to produce various effects in the ambient plasma that modify the Langmuir sheath (see Alpert et al., 1965), and some of them propagate along the magnetic field forming "Alfvén wings". Kinetic models of these effects begin to appear in the literature (see for example, Singh et al., 1994), however at this stage they are still hardly applicable for our primary purpose of calculating the trajectories and energies of ions entering the aperture of a plasma instrument. Further modelling work is in progress in which the outer boundary of the sheath is specially chosen to be at a finite distance for each particular Debye length $D$.

\section{The instrument RON-AC for active potential control}

\subsection{Purpose of the experiment}

The main objective of the spacecraft potential control experiment (RON-AC) is to neutralise and thereby stabilise the positive floating potential which a sunlit spacecraft often acquires. Such conditions in near-Earth space are met outside the plasmasphere, where the plasma density is low and as long as the electron temperature is not too high (less than a few $\mathrm{keV}$ ). Observed spacecraft potentials range from a few Volts to a few tens of Volts positive. Such potentials may 


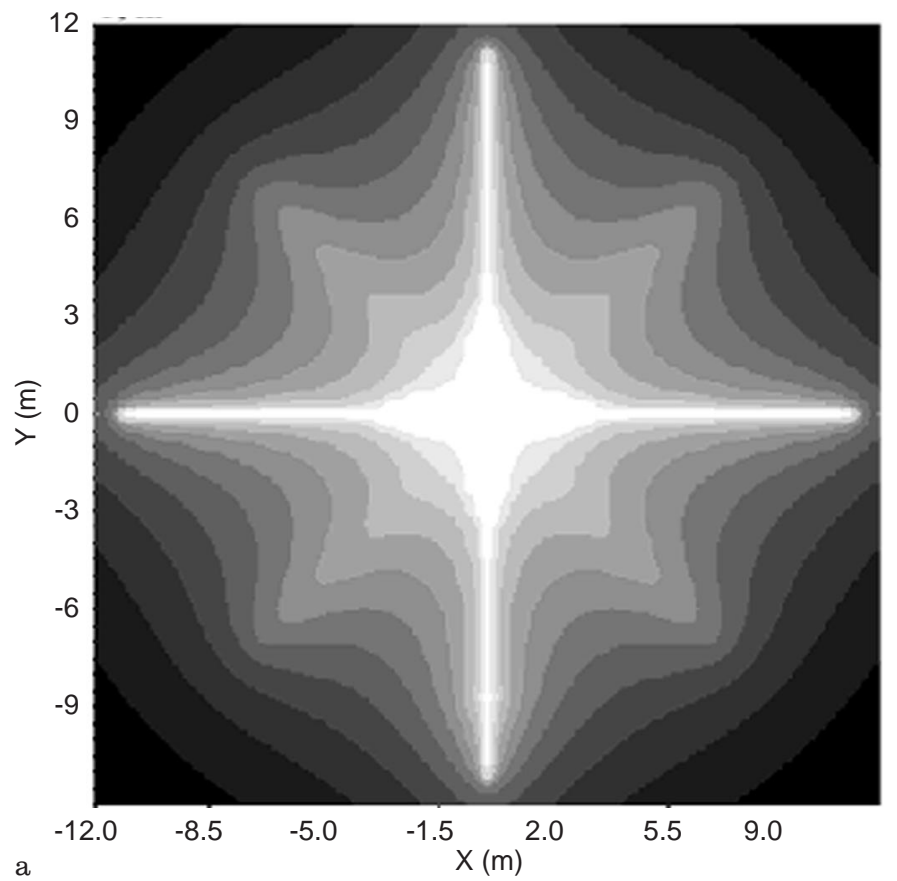

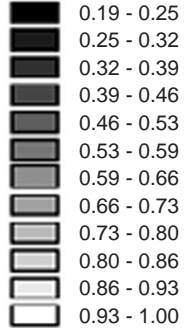

Solar panels and bars

$Z=0$
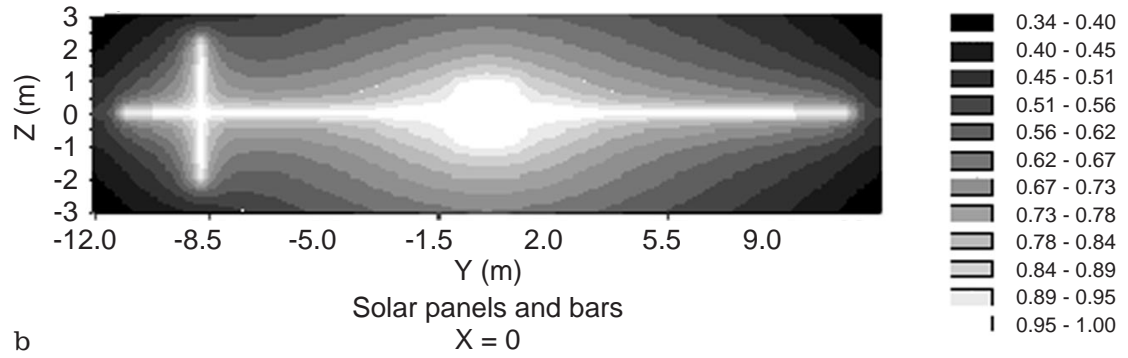

significantly distort measurements of the thermal plasma ion distribution functions and so present an important source of error for ion mass spectrometry from a high altitude satellite (see Sect. 5). As can be seen in Sect. 4, ion currents of up to a few tens of $\mu \mathrm{A}$ fulfil the objectives of the experiment for the conditions on-board of INTERBALL-2. The instrumental set-up generating these currents is briefly outlined below. A more comprehensive description has been given by Riedler et al. (1995).

\subsection{Technical implementation}

The instrument hosts two different and independent ion beam systems which can provide an adjustable current of singly charged indium or $\mathrm{N}_{2}^{+}$ions. The instrument is a single mechanical unit consisting of the electronics box and the two ion sources with auxiliary systems, mainly a gas flow control system for the nitrogen source, mounted on top. Figure 3 shows the instrument with its carbon fibre dome being removed, which protects the ion sources and the gas system in flight configuration. The instrument is accommodated on a platform pointing towards the Sun. The ion beams are ejected in upward, i.e. sunward direction, parallel to the spacecraft spin axis. This configuration had been chosen to avoid
Fig. 2a,b. Three-dimensional electric field distribution around INTERBALL-2: $\mathbf{a}$ in the plane of the solar panels, $\mathbf{b}$ in the plane of "the spacecraft meridian" through the electric field booms

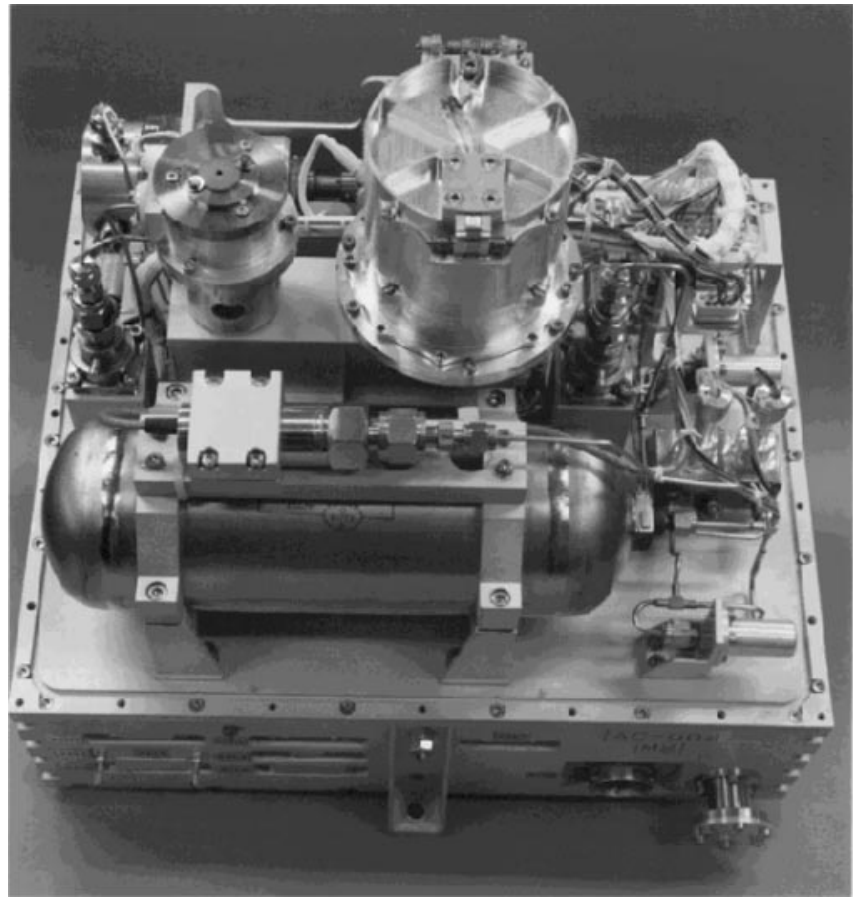

Fig. 3. The instrument RON-AC with the ion emitter modules and the gas flow control system 
any modulation effects by a beam rotating with the spacecraft.

3.2.1 Liquid metal ion source. This ion emitter is a "solid needle" - type liquid metal ion source using indium as charge material. A solid needle made of tungsten with a tip radius between 2 and $15 \mu \mathrm{m}$ is mounted in a heated reservoir with the molten indium as the charge material. A potential of 5-7 kV, applied between the needle and an extractor electrode, pulls the liquid metal towards the extractor electrode, which forms a Taylor-cone with an apex diameter of a few nanometres. Field evaporation of positively charged metal atoms in the strong apex field leads to the emission of an ion beam. The focusing lens shapes the beam to a finite divergence of about $15^{\circ}$ (full width half maximum). Single tips, as used in this instrument and previously on the GEOTAIL spacecraft (Schmidt et al., 1993) can produce currents of several tens of $\mu \mathrm{A}$. The mass efficiency is high. One gram of indium suffices for about $10000 \mathrm{~h}$ of continuous operation at 10 to $15 \mu \mathrm{A}$. Indium has been chosen as the charge material because of its low vapour pressure, which prevents contamination of nearby surfaces, and its non-toxic properties.

For reasons of lifetime and redundancy, four individual emitters are mounted in a common system, with only one emitter being active at a time. The active source is heated by its individual electric heater. Only about $0.5 \mathrm{~W}$ are required for this purpose. This low figure is the result of the small dimensions and the extremely good thermal isolation of the emitters by a slab of porous ceramic material.

In order to avoid oxidation of the indium it should never become exposed to air or water vapour. Therefore the emitters were encapsulated in an almost hermetically closed container which was opened after launch.

Unfortunately, an early failure of the high-voltage supply prevented the operation of these emitters on INTERBALL-2. As the instrument was conceived from the beginning as a technological endeavour, essential parts, such as the supplies and the ion sources, were redundant. The second type of ion source and its supply are described below.

3.2.2 Saddle field ion source. The saddle field ion source consists of a cylindrical grounded tube of about $40 \mathrm{~mm}$ diameter and $30 \mathrm{~mm}$ length, that accommodates in its centre a ring-shaped anode connected to a positive potential of up to $6 \mathrm{keV}$. The particular shape of the internal electric field configuration causes electrons to describe longer oscillatory trajectories and increases the ionisation probability. Two symmetrical ion beams can be extracted from the flat sides of the cylinder through small apertures. This design utilises one beam. The other beam hits a plate mounted at an angle of $45^{\circ}$ as part of the ion catcher. The electrical efficiency of the this ion source amounts to a few per cent only, but no additional heater elements are required. The system requires an elaborate gas flow control system to establish a controllable and very small flow of nitrogen gas into the ion source.

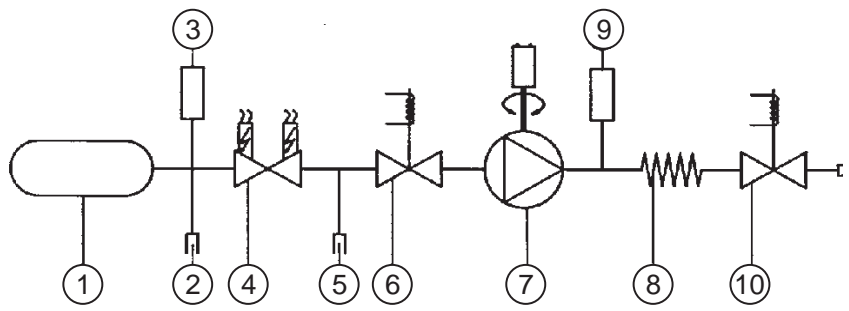

Fig. 4. Gas flow control system: (1) gas bottle, (2) filling nipple, (3) pressure transducer, (4) pyro valve, (5) test port connection, (6) latching valve, (7) motor-driven pressure regulator, (8) constant leak, (9) pressure transducer, (10) magnetic latching-valve

3.2.3 Gas flow control system. A schematic of the gas flow control system is shown in Fig. 4. The gas bottle (1) contains ultra pure nitrogen under high pressure (18 bar) and has a volume of 0.5 litres. This bottle can be filled through a nipple (2). The gas pressure is measured by pressure transducer (3) with a measuring range of up to 35 bar absolute. The gas is fed from the bottle to the pyrotechnic valve (4) which secures a hermetically sealed volume of gas. The valve can be opened by activating a pyrotechnic piston actuator. The gas flow control system and its mechanisms have been described in detail elsewhere (Arends and Scheper, 1989; Arends and Gonfalone, 1976; Gonfalone and Arends, 1979).

When the pyrotechnic valve is open the gas flows to a magnetic latching valve (6) and thereafter to the pressure regulator (7), which reduces the high primary pressure to a very constant lower secondary pressure. The setting of the regulator can be changed by a DC motor. The secondary pressure remains constant nearly independent of the value of the primary pressure. The secondary gas pressure is measured by a pressure transducer (9). The secondary side of the pressure regulator is further connected to a constant impedance (8) with a leak rate of $6 \times 10^{-4} \mathrm{mb}$ litre $\mathrm{s}^{-1}$ at $1000 \mathrm{mb}$ for air. After this impedance the gas flows to a magnetic latching valve (10), which controls the gas flow directly at the entrance to the saddle field ion emitter. All the different components of the system are interconnected by stainless steel capillaries.

As the gas flow of the system is very small depending on the working mode of the source between 4.5 and $8 \times 10^{-4} \mathrm{mb}$ litre $\mathrm{s}^{-1}$ - the characteristics of the source mainly depend on the stability of the pressure regulator. The photograph in Fig. 4 shows the implementation of the gas flow control system (protective dome removed). The large cylinder in the centre is the liquid metal ion source module (cover in closed position), the smaller one to the left is the saddle field ion source (with the small aperture of $1 \mathrm{~mm}$ in diametre for the ion beam in the centre). The emitters are surrounded by components of the gas flow control system: The latching valves $(6,10)$ are mounted immediately to the left and right of the ion sources. The motor-driven pressure regulator (7) is partly hidden behind the saddle field ion source. The gas bottle (1) in front is topped by one of the pressure transducers (3). Further to the right 
of the bottle the housings of the redundant pyrotechnic piston actuators (4) are visible. The main filling nipple (2) is located at the lower right corner of the platform.

3.2.4 Control electronics. The instrument utilises a microprocessor for controlling the experiment and for data handling. It basically operates and controls the ion emitter and gas flow control systems, performs the startup procedure of the emitters, and serves the interfaces to the spacecraft telemetry systems, telecommand and the experiment IESP-2M (Perraut et al., this issue), which provides spacecraft potential data to the instrument RON in real-time. Special attention is paid to the monitoring of the high voltage unit and the measurement of the effective ion beam current, which necessitates a special grounding concept for the emitter supply unit.

\section{Some results of the on-board measurements}

Several instruments are capable of measuring the electric potential on-board the satellite INTERBALL-2:

1. KM-7 (electron temperature detector)

2. ALFA-3 (thermal plasma ion flux detector - a modulation ion trap)

3. IESP-2 M (DC electric field and ULF wave detector)

Apart from these, the responses of the energy-massangle ion spectrometer HYPERBOLOID, and of the low-energy particle spectrometers SKA-3, ION and PROMICS, are influenced, at their low-energy range, by the momentary value of $V_{\text {sat }}$. At this stage, no intercomparisons of these data have been performed, as only very preliminary data on $V_{\text {sat }}$ are available now. The detailed analysis of the measured spacecraft potential will be reported in a later paper.

Preliminary evaluations, however, show that $V_{\text {sat }}$ usually was between 0 and $6 \mathrm{~V}$, mostly about 3 to $4 \mathrm{~V}$ positive. A typical example of the variations of the spacecraft potential with and without active ion beam emission is shown in Fig. 5. The time interval covers 100 min of a pass over the auroral region (on 20 October, 1996, at 21:40-23:00 UT). The plasma density at that time remains to be analysed. The lower panel shows the current of the nitrogen ion source of RON. The emitter is turned on at $21: 45$ UT with $8 \mu \mathrm{A}$. The beam current is increased three times up to about $12.5 \mu \mathrm{A}$ and turned off at 22:17 UT. The upper panel shows the spacecraft potential as received from the instrument IESP-2M (Perraut et al., this issue) via the on-board interexperiment link. Note that this panel shows the voltage difference between the probes and the spacecraft body rather than the true potential of the spacecraft relative to the ambient plasma. The true potential is obtained by adding the floating potential of the probes, which is kept at a low value by enforcing an electron current into the plasma, but nevertheless is of the order of 1 to 2 Volts positive. After a few initial adjustments the instrument IESP-2M enters its normal operational condition within the first current step and measures a very low and almost constant potential of $2 \mathrm{~V}$. Notably the potential remains at this value independent of the ion beam current. Only a very faint decrease can be seen during the last current

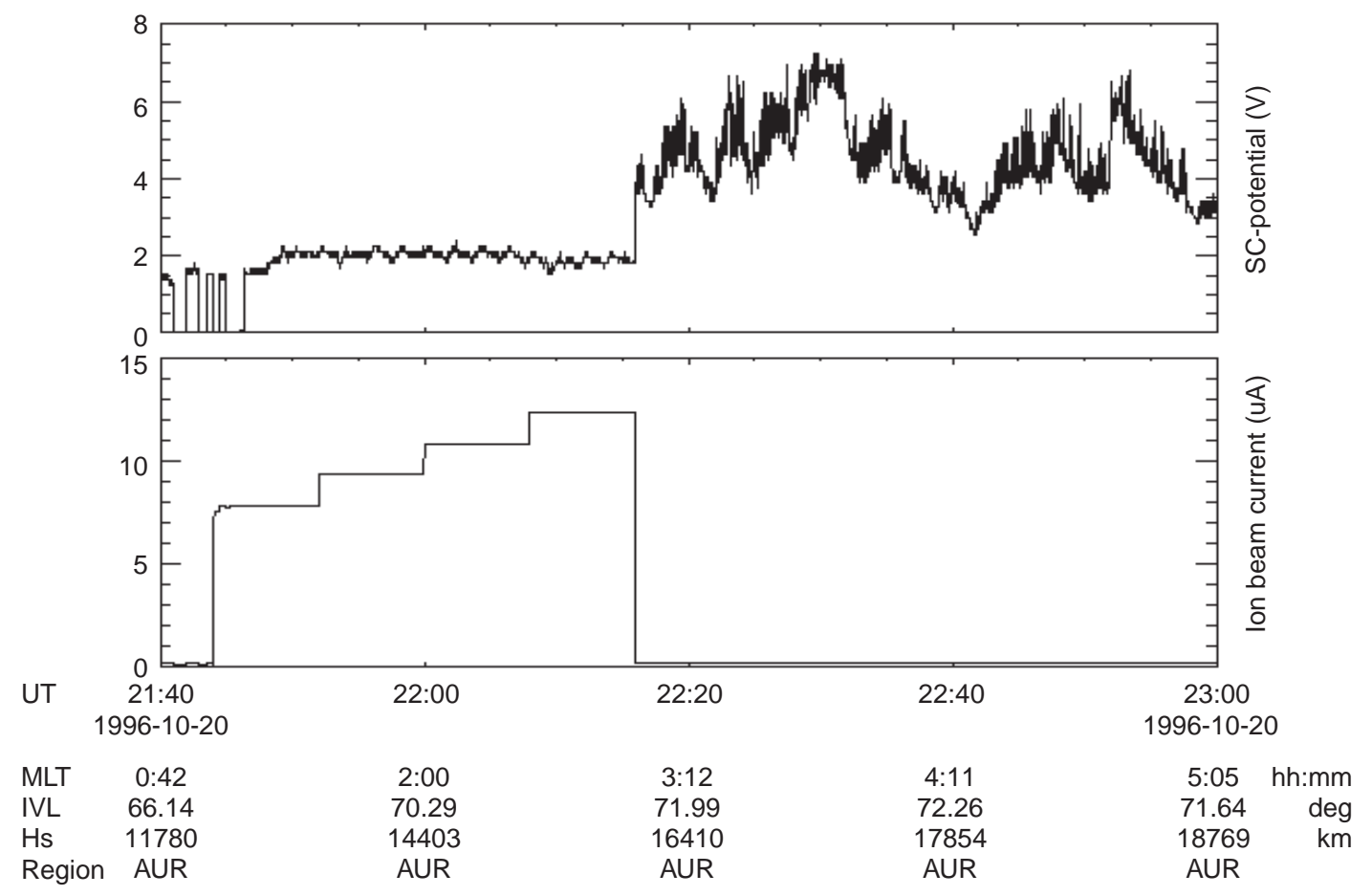

Fig. 5. Effect of active ion beam emission on the spacecraft potential, measured on 20 October, 1996, at 21:40-23:00 UT; upper panel: spacecraft potential data received from the instrument IESP-2M via the on-board inter-experiment link; lower panel: ion beam current 
step. At close inspection this decrease turns out to occur only after the change of the current. Therefore it is likely to be related to a change in ambient plasma parameters rather than being directly related to the emission current. The conclusion to be drawn is that currents between 8 and $12.5 \mu \mathrm{A}$ have the same effects at the accuracy provided by the present set-up.

After the ion emission has been turned off, the picture changes dramatically. The potential starts to fluctuate between 3 and $7 \mathrm{~V}$, and very fast changes are superposed on variations with a time scale of minutes. With the spacecraft potential of the INTERBALL-2 satellite not being extremely high, the stabilisation of the potential by active ion beam emission is the most advantageous effect, supporting the fine-tuning of low-energy plasma instruments to the ambient plasma conditions and the interpretation of the data.

Figure 6 shows another example of the data on 29 October, 1996. Before 21:00 UT and after 21:30 UT the potential is again clamped to an almost constant value due to the simultaneous operation of the ion beam at $9.7 \mu \mathrm{A}$. Immediately after the ion beam has been turned off at 21:00, the potential starts to fluctuate as in the previous example. The new feature in Fig. 7 is the gradual decrease of the uncontrolled potential towards a value stabilising slightly above $2 \mathrm{~V}$. This decrease is accompanied by an increase of the flux of energetic electrons and ions measured by the instruments ION and HYPERBOLOID (Sauvaud, private communication; Dubouloz, private communication). The effects of plasma density and temperature on the spacecraft potential are clearly illustrated, as well as the fact that the effect of the ion beam is more pronounced at low density.

\section{Current balance at the spacecraft surface}

\subsection{The general case}

The basic condition for the equilibrium spacecraft potential $V_{\text {sat }}$ of a satellite is that $I_{\mathrm{T}}\left(V_{\text {sat }}\right)=0$, where $I_{\mathrm{T}}$ is the total current to satellite surface. In the general case (Garrett, 1981, eq. 10) this relation can be written as:

$I_{\mathrm{e}}-\left[I_{\mathrm{i}}+I_{\mathrm{se}}+I_{\mathrm{si}}+I_{\mathrm{bse}}+I_{\mathrm{ph}}\right]+I_{\mathrm{b}}=0$

where

$I_{\mathrm{e}}$ electron current incident on the satellite surface

$I_{\mathrm{i}}$ ion current incident on the satellite surface

$I_{\mathrm{se}}$ secondary electron current resulting from $I_{\mathrm{e}}$

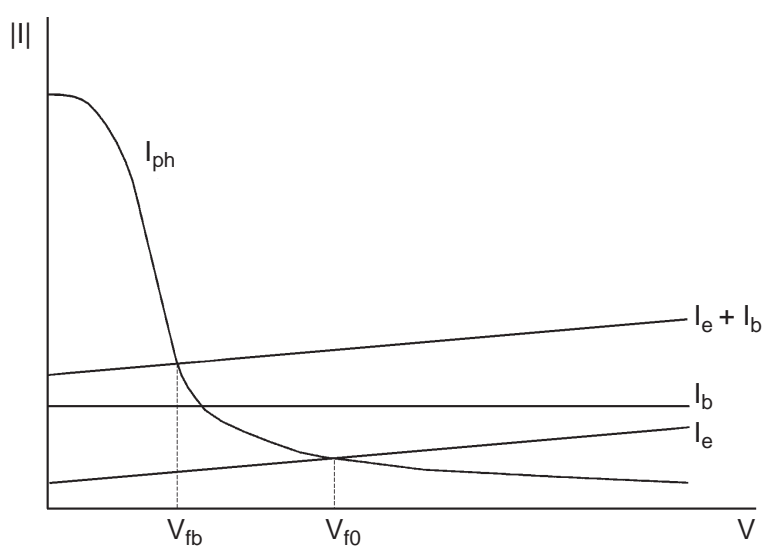

Fig. 7. Representative spacecraft current-voltage characteristics, showing the effect of active ion emission on the spacecraft potential

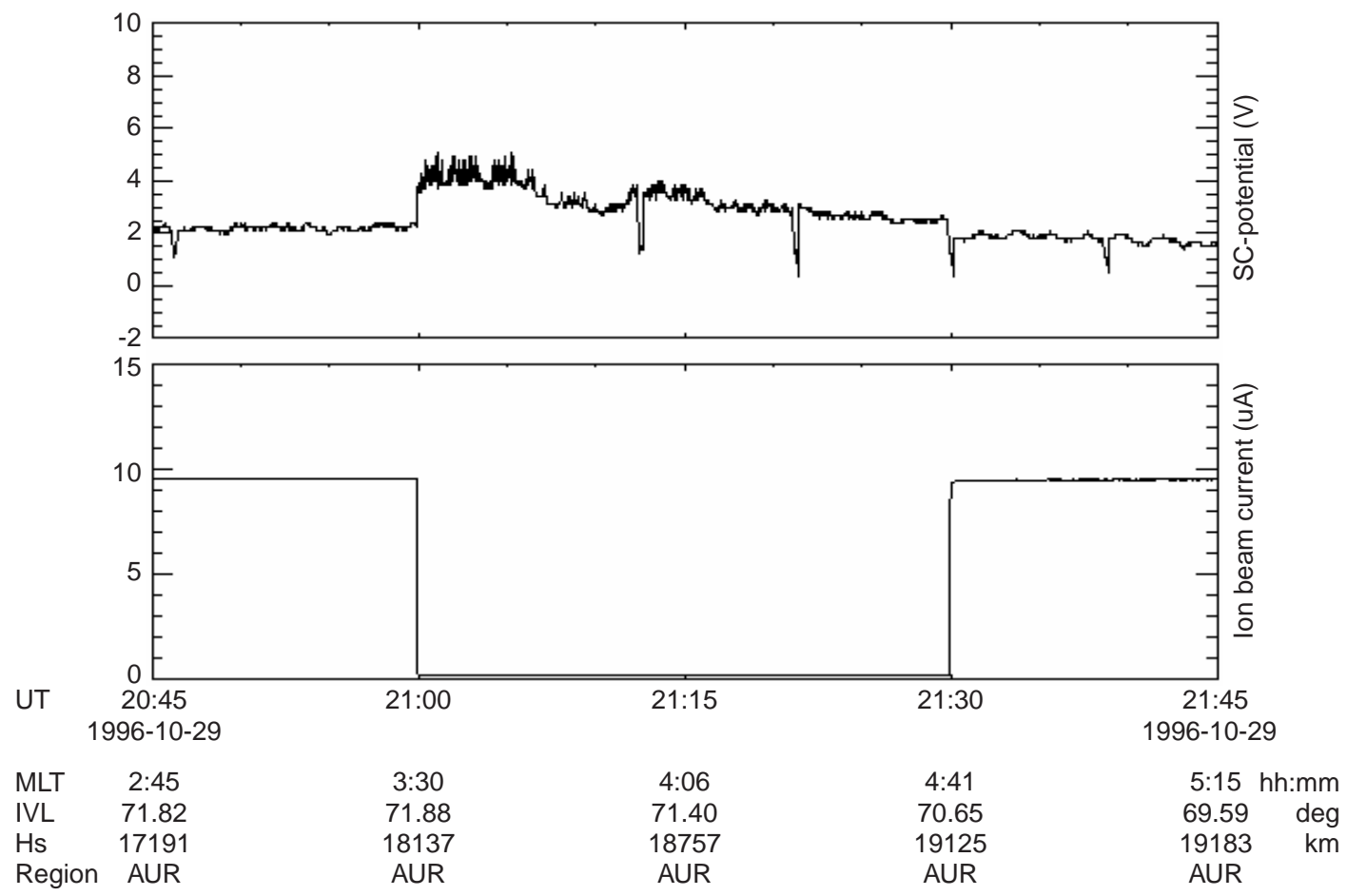

Fig. 6. Same as Fig. 5, but for 29 October, 1996, 20:45-21:45 UT. The five short drop-outs in the potential are instrumental artefacts 
$I_{\mathrm{si}}$ secondary electron current resulting from $I_{\mathrm{i}}$

$I_{\mathrm{bse}}$ current of backscattering electrons resulting from

$I_{\mathrm{ph}}$ photo-electron current

$I_{\mathrm{b} 1}$ 1current from active on-board ion sources

All currents are functions of $V_{\text {sat }}$. However, the dependence of $I_{\mathrm{b}}$ on $V_{\text {sat }}$ can be safely neglected if the acceleration voltage of the particle beam is much larger than $V_{\text {sat }}$.

As the observations of the spacecraft potential for INTERBALL-2 show positive values, we shall analyse the contribution of each component of Eq. (3) assuming $V_{\text {sat }}>0$. To simplify the analysis we shall further assume plasma neutrality $\left(n_{\mathrm{e} 0}=n_{\mathrm{i} 0}=n_{0}\right)$ with $n_{\mathrm{e} 0}$ and $n_{\mathrm{i} 0}$ being the undisturbed plasma electron and ion densities outside the sheath, respectively.

\subsection{Photo-electron current}

The photo-electron current $I_{\mathrm{ph}}$ constitutes the main factor causing a positive spacecraft potential. As the spin axis of the INTERBALL satellites points towards the Sun, the large area of the four solar panels covered by indium oxide $\left(2.25 \mathrm{~m}^{2} \times 4=9.0 \mathrm{~m}^{2}\right)$ becomes the main source of photo-electrons. While their efficiency for photo-electron emission under solar illumination has not been measured yet, there is ample reason to assume that the well-established photo-electron production coefficient for indium oxide $\left(30 \mu \mathrm{A} \mathrm{m}^{-2}\right.$, see Table 4 in Garrett, 1981) may be used, at least as a first approximation. The solar side of the satellite body adds another area of $4.15 \mathrm{~m}^{2}$ which is partly covered by gold-metallised mylar and partly by indium oxide. We take for these surfaces the average of the respective values in the same Table 4 in Garrett (1981). Thus, the total photo-electron current $I_{\mathrm{ph} 0}$ is estimated as $(9.0 \times 30)+4.15 \times(29+30) / 2=0.392 \mathrm{~mA}$, i.e. the mean photo-electron current density results in $J_{\mathrm{ph} 0}=29.8 \mu \mathrm{A} \mathrm{m}^{-2}$.

Under the conditions of a rarefied plasma, when $\left|I_{\mathrm{ph}}\right|$ exceeds all other currents into or from the ambient plasma, the spacecraft potential adjusts itself at a level where most of the photo-electrons are reflected back to the surface or trapped within the sheath, and only the high energy tail of the photo-electrons can escape into the ambient plasma and establish an equilibrium with the other currents. It may be speculated that the spectrum of the solar emissions, in particular the intensity in the EUV range and of X-rays, affects the production rate and the energy spectrum of the photo-electrons. It is assumed that during the deep solar minimum conditions prevailing now, the solar intensity of solar X-rays and EUV is low, and so is the photo-electron production rate. If the energy spectrum of the photo-electrons is approximated by a Maxwellian distribution, the current of escaping photo-electrons $I_{\mathrm{ph} 0}$ can be represented as

$I_{\mathrm{ph}}=A_{\mathrm{S}} J_{\mathrm{ph} 0} \exp \left\{-q \frac{V_{\mathrm{sat}}}{k T_{\mathrm{ph}}}\right\}$, for $V_{\mathrm{sat}}>0$ where $q$ is the elementary charge, $k T_{\mathrm{ph}} / q$ is the average energy of photo-electrons, and $A_{\mathrm{S}}$ is the sunlit area of the satellite surface.

\subsection{Plasma electron and ion currents on the satellite surface}

To estimate the contribution of the electron and ion currents from the ambient plasma to the current balance of the INTERBALL-2 satellite we shall refer to equations $(22,23)$ from Garrett (1981) for the case of positive spacecraft potential:

$$
\begin{aligned}
& I_{\mathrm{e}}=I_{\mathrm{e} 0}\left(1+q \frac{V_{\mathrm{sat}}}{k T_{\mathrm{e}}}\right) \\
& I_{\mathrm{i}}=I_{\mathrm{i} 0} \exp \left\{-q \frac{V_{\mathrm{sat}}}{k T_{\mathrm{i}}}\right\}
\end{aligned}
$$

where

$I_{\mathrm{e}, \mathrm{i} 0}=A_{\mathrm{T}} J_{\mathrm{e}, \mathrm{i} 0}=A_{T} \frac{q n_{0}}{2}\left(\frac{2 k T_{\mathrm{e}, \mathrm{i}}}{\pi m_{\mathrm{e}, \mathrm{i}}}\right)^{1 / 2}$

where $A_{\mathrm{T}}$ is the total surface area of the satellite. It is here assumed that the satellite velocity $v_{\mathrm{s}}$ is less than the thermal ion velocity $v_{i}$, so the processes related to the satellite wake for ions are not considered, for example in ion outflows. In a simple calculation we obtain the value of $J_{\mathrm{e} 0}$ :

$J_{\mathrm{e} 0}=26.8 n_{0}\left(k T_{\mathrm{e}} / q\right)^{\frac{1}{2}} \mathrm{nA} \mathrm{m}^{-2}$

where the undisturbed plasma density $n_{0}$ is expressed in $\mathrm{cm}^{-3}$ and the energy of the plasma electrons, $k T_{\mathrm{e}} / q$, in $\mathrm{eV}$. For typical parameters of the main part of the INTERBALL-2 orbit $\left(n_{0} \approx 1 \ldots 100 \mathrm{~cm}^{-3}, \quad k T_{\mathrm{e}} /\right.$ $q \approx 1 \mathrm{eV}) J_{\mathrm{e} 0}$ is at least one order of magnitude lower than $J_{\mathrm{ph} 0}$ estimated.

Let us now consider the incident ion current $I_{\mathrm{i}}$. It can be seen that even for $V_{\text {sat }}=0$, and thermal equilibrium between electrons and ions, the ratio $I_{\mathrm{i}} / I_{\mathrm{e}}$ corresponds to the ratio between the ion and electron thermal velocities, i.e. it is very small. With increasing $V_{\text {sat }}$ this ratio becomes even smaller, which means that for a positively charged spacecraft the incident ion current may be neglected in the current balance.

\subsection{The average secondary electron emission coefficient for INTERBALL-2}

In order to estimate the influence of the secondary electron emission on the charging of the INTERBALL2 satellite, let us assume a Maxwellian energy distribution for the energetic part of the secondary electron spectra:

$I_{\mathrm{se}}=\sigma\left(T_{\mathrm{e}}\right) I_{e 0} \exp \left\{-q \frac{V_{\mathrm{sat}}}{k T_{\mathrm{se}}}\right\}, \quad$ for $V_{\mathrm{sat}}>0$ 
The secondary electron emission coefficient $\sigma$ is about zero for $1 \mathrm{eV}$ primary electrons, but increases to $0.8 \ldots 0.9$ or more for energies of $100 \mathrm{eV}$ to $1 \mathrm{keV}$, and the average energy of secondary electrons $k T_{\mathrm{se}} / q$ is typically a few eV (Whipple, 1965). In order to become significant for the current balance $I_{\mathrm{se}}$ would have to become comparable to $I_{\mathrm{ph}}$, which would require densities $n_{\mathrm{e} 0} \approx 200$ to $300 \mathrm{~cm}^{-3}$ at energies of $100 \mathrm{eV}$ to $1 \mathrm{keV}$, where $\sigma$ increases. Such values do not occur at the orbit of INTERBALL-2. Thus, secondary electron emission can be neglected for positive potentials of the satellite INTERBALL-2. We also can neglect backscattering of electrons and secondary electrons due to the impact of ions, because the corresponding coefficients are even lower than the secondary electron emission coefficient.

\subsection{Principle of spacecraft potential control}

The basic approach of this method of active spacecraft potential control is illustrated by Fig. 7. Without active ion emission, the equilibrium potential adjusts itself at a high positive value $V_{\mathrm{f} 0}$ corresponding to the equilibrium between the high energy tail of the emitted photoelectrons $\left(I_{\mathrm{ph}}\right)$ and the plasma electron current $I_{\mathrm{e}}$. By adding a positive ion beam current $I_{\mathrm{b}}$, the potential is quickly reduced to a value $V_{\mathrm{fb}}$, which is of the order of the mean energy of the photo-electrons, even if the emitted ion current remains well below the total photoemission current. It is also obvious that the potential in the uncontrolled case results from an intersection between the photo-electron and plasma electron currents at a shallow angle, and therefore is very sensitive to variations of the plasma conditions. This dependence, which results in a large variance of the floating potential, is highly reduced by the addition of a constant beam current.

\subsection{Plasma conditions for positive spacecraft potential and active potential control at the INTERBALL-2 satellite}

Now we shall estimate where along the orbit of INTERBALL-2 positive spacecraft potentials can be expected. On the satellite, the ion beam emitter RON was used as an active current source with ion energies of 4 to $5 \mathrm{keV}$ (see Sect. 3). Using Eqs. $(4,5)$ Eq. (3) can be written as $I_{\mathrm{e}}-I_{\mathrm{ph}}+I_{\mathrm{b}}=0$ or:

$$
A_{\mathrm{T}} J_{\mathrm{e} 0}\left(1+q \frac{V_{\mathrm{sat}}}{k T_{\mathrm{e}}}\right)-A_{\mathrm{S}} J_{\mathrm{ph} 0} \exp \left\{-q \frac{V_{\mathrm{sat}}}{k T_{\mathrm{ph}}}\right\}+I_{\mathrm{b}}=0
$$

which can easily be solved for $V_{\text {sat }}$ by numerical methods. Some results, using parameters of INTERBALL-2 $\left(A_{\mathrm{S}}=13.15 \mathrm{~m}^{2}, A_{\mathrm{T}} \approx 40 \mathrm{~m}^{2}\right)$, and assuming a mean photo-electron energy of $k T_{\mathrm{ph}} / q=1.5 \mathrm{eV}$, are compiled in Table 1. This choice of $T_{\mathrm{ph}}$ is supposed to model the main part of the photoelectrons, mainly generated by solar Lyman alpha radiation, but does not include any correction for a more energetic component.

Table 1 shows basic agreement between the calculated spacecraft potential therein and the measurements (see Fig. 5). The floating potential without ion beam ranges between about 2 and $6 \mathrm{~V}$ for the typical plasma conditions along the orbit of INTERBALL-2 (the two upper rows in Table 1). When the plasma is more rarefied, a condition which may occasionally be fulfilled on this orbit, but is more likely in the outer magnetosphere, the potential increases. When the ion beam is turned on, the potential becomes independent of the density for densities below $\approx 1 \mathrm{~cm}^{-3}$ (for $20 \mu \mathrm{A}$ ion current). In this regime the current carried by the plasma electrons becomes negligible relative to the currents of the photo-electron and the ion beam, and the equilibrium potential is determined by the ion beam current. The potential varies only by 1 or 2 Volts even for largely different (some tens of $\mu \mathrm{A}$ ) ion currents. As secondary electron production can be ignored in our case (as is done in the calculations for Table 1) the influence of the plasma temperature is small.

\section{Models of thermal plasma distortions around INTERBALL-2 for positive spacecraft potential}

\subsection{Some model results for infinite Debye length}

Comprehensive measurements of the three-dimensional distribution functions of thermal and suprathermal ions $\mathrm{H}^{+}, \mathrm{He}^{+}, \mathrm{O}^{+}$and $\mathrm{O}^{++}$ions are being carried out onboard INTERBALL-2 by the energy-mass-angle spectrometer HYPERBOLOID (see the accompanying work by Dubouloz et al., this issue). Also, low energy particle measurements are carried out on-board by several other instruments. Calculations using the infinite Debye length model I2 S of Sect. 2.3 were performed to simulate the responses of the HYPERBOLOID instru-
Table 1. Spacecraft potential calculated for different ambient plasma parameters

\begin{tabular}{lllll}
\hline $\begin{array}{l}\text { Plasma density } \\
\left(\mathrm{cm}^{-3}\right)\end{array}$ & $\begin{array}{l}\text { Plasma electron } \\
\text { energy }(\mathrm{eV})\end{array}$ & $\begin{array}{l}\text { Spacecraft } \\
\text { potential }(\mathrm{V}) \\
\text { for } I_{\text {beam }}=0 \mu \mathrm{A}\end{array}$ & $\begin{array}{l}\text { Spacecraft } \\
\text { potential }(\mathrm{V}) \\
\text { for } I_{\text {beam }}=12.5 \mu \mathrm{A}\end{array}$ & $\begin{array}{l}\text { Spacecraft } \\
\text { potential }(\mathrm{V}) \\
\text { for } I_{\text {beam }}=20 \mu \mathrm{A}\end{array}$ \\
\hline 30 & 0.3 & 1.77 & 1.68 & 1.63 \\
1 & 1 & 5.96 & 4.59 & 4.11 \\
0.1 & 1 & 8.88 & 5.10 & 4.43 \\
0.01 & 1 & 11.93 & 5.17 & 4.46 \\
0.001 & 1 & 15.06 & 5.18 & 4.47 \\
\hline
\end{tabular}


ment to anisotropic (bi-Maxwellian) ion distribution functions for different spacecraft potentials and initial flow velocities $\vec{U}$ of the ions in a magnetic coordinate system moving with the the spacecraft. (The velocity $\vec{U}$ of the ions, in the spacecraft frame, results from the sum of the negative satellite's velocity and the velocity of the ion flux with respect to the magnetic field). $\varepsilon$ is the angle between the magnetic field and the velocity vector $\vec{U}$.

Figure $8 \mathrm{a}-\mathrm{d}$ shows some results of the model calculations and contain contours of equal flux, labelled with the logarithm (base 10) of the flux in $\mathrm{cm}^{-2} \mathrm{~s}^{-1}$, as a function of the velocity components of the ions parallel and perpendicular to the magnetic field. The plane shown contains the vectors of the magnetic field and the velocity $\vec{U}$. The bi-Maxwellian distribution function used as an input corresponds to the following set of parameters: $T_{\text {par }}=10000 \mathrm{~K}, T_{\text {perp }}=20000 \mathrm{~K}$, and the ion density is $5 \mathrm{~cm}^{-3}$. The flow velocity $\vec{U}$ in the magnetic frame moving with the satellite is assumed to be $10 \mathrm{~km} \mathrm{~s}^{-1}$ at $\varepsilon=60^{\circ}$. Figure a-d refers to increasing satellite potentials $V_{\text {sat }}$ of $0,5,10$, and $15 \mathrm{~V}$, respectively. It can be seen that the distortions of the shape of the measured ion distribution functions due to the ion trajectories passing through the potential distribution around the satellite can be significant. Already at $0 \mathrm{~V}$ the originally bi-Maxwellian distribution is shifted according to the flow velocity. Contours near $\vec{U}=0$, where the flux as "seen" by an instrument on the spacecraft vanishes, are not shown. With increasingly positive satellite potential the measured fluxes decrease,
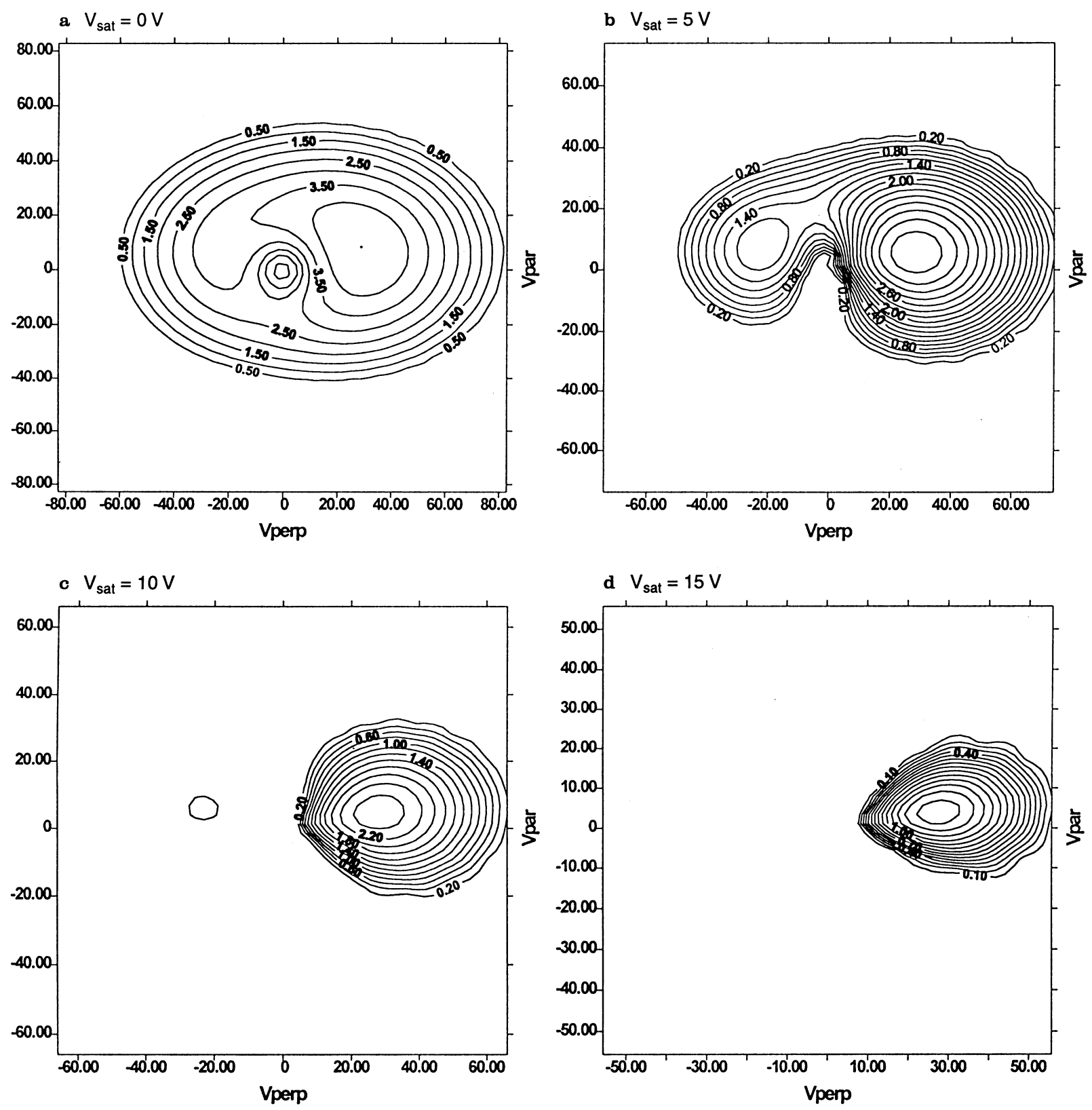

Fig. 8a-d. Some results of the model calculations for ion distribution functions (see text); $v_{\text {par }}$ and $v_{\text {perp }}$ denote the velocity components parallel and perpendicular to the magnetic field in $\mathrm{km} \mathrm{s}^{-1}$. Labels are in $\log _{10}$ of the flux in $\mathrm{cm}^{-2} \mathrm{~s}^{-1}$ 
and distortions become more pronounced. Finally, the flux distribution assumes a beam-like shape in Fig. 8d. Fortunately, the preliminary evaluations show that the spacecraft potential was rather low for a significant part of the flight, between nearly zero and 4 to $6 \mathrm{~V}$ positive, but even in this range the sheath effects should not be neglected.

\subsection{Modification of the ion detector response due to the potential at the aperture grid}

In the case of a significant positive spacecraft potential $V_{\text {sat }}$ and a large Debye length $D$ in a rarefied plasma, only ions with the energy $E_{\mathrm{i}}>V_{\text {sat }}$ in the spacecraft frame can reach the detector, and their energy will be reduced accordingly. Thus, setting the aperture grid at the entrance of an instrument, such as the HYPERBOLOID spectrometer, to a negative potential allows the detector to collect and accelerate ions with initial energy $E_{\mathrm{i}}>V_{\text {sat }}$ to the spectrometer aperture and thus increase the sensitivity, but the ions are still affected by the potential well around the satellite. Of course, this very crude analysis does not take into account inhomogeneities in the potential associated with the complex shape of the satellite. Even with RON being operational, it is expected that $V_{\text {sat }}$ remains slightly positive. The usual setting of the bias voltage $V_{\text {bias }}$ is -6 to $-8 \mathrm{~V}$, so that $V_{\mathrm{HYP}}=V_{\text {sat }}+V_{\text {bias }}$ remains close to zero. The value of $V_{\text {HYP }}$ has to be taken into account in any quantitative analysis of HYPERBOLOID data.

\section{Conclusions}

The metallised and practically equipotential surfaces of the INTERBALL-2 satellite are the key factor leading to relatively low positive spacecraft potentials observed at high altitude. Ion beam currents of up to tens of microamperes are needed for the active control of the positive spacecraft potential. The RON-AC ion beam emitter was capable of providing such ion currents in flight and allowed to perform experiments on the active control of the spacecraft potential.

Calculations were performed to model the ion trajectories around a realistic model of the INTERBALL-2 satellite in a static three-dimensional electric field distribution for infinite Debye length, and neglecting magnetic fields. From these calculations the response of the on-board energy-mass-angle thermal ion spectrometer HYPERBOLOID for initial anisotropic (biMaxwellian) ion distribution functions was calculated, using realistic values of the spacecraft potential. It is shown that significant distortions of the measured ion distribution functions are possible, especially in the presence of strongly anisotropic distributions.
Acknowledgements. The authors wish to thank O. Akentieva, P. Marrama, M. Mogilevsky, and J. Smilauer for their generous contributions to this work and sharing of their data. DVC, LVZ, MVV, TMM and YIG thank INTAS for the grant 94-1695 and the RFBB for the grant 97-02-6333.

Topical Editor K.-H. Glassmeier thanks N. Dubouloz and another referee for their help in evaluating this paper.

\section{References}

Alpert, Y. L., A. V. Gurevich, and L. P. Pitaevsky, Artificial satellites in a rarefied plasma, NUKA Publishing House, Moscow, 1965.

Arends, H. J., and A. A. Gonfalone, Mechanical opening system for vacuum tubes in space environment, Rev. Sci. Inst., 47, 153-155, 1976.

Arends, H. J., and R. Scheper, Gasflow control system for very low gasflows to maintain a constant pressure in a saddle-field ion source, Int. Rep., SSD of ESA/ESTEC, Noordwijk, The Netherlands, 1989.

Brace, L.H., W.R. Hoegy and F. Theis, Solar EUV measurements at Venus based on photoelectron emission from the Pioneer Venus Langmuir probe, J. Geophys. Res., 93, 7282-7296, 1988.

Dubouloz, N. et al., this issue

Garrett, H. B., The charging of spacecraft surfaces, Rev. Geophys. Space Phys., 19 (4), 577-616, 1981.

Gonfalone, A. A., and H. J. Arends, Open vacuum tube in space, Rev. Sci. Inst., 50, 1484-1485, 1979.

Laakso, H., and A. Pedersen, Satellite photoemission characteristics, in Materials in a Space Environment, Ed. H.T.D. Guyenne, ESA SP-368, 361-365, 1994.

Pedersen, A., Solar wind and magnetosphere plasma diagnostics by spacecraft electrostatic potential measurements, Ann. Geophysicae, 13, 118-129, 1995.

Perraut, S. et al., this issue

Riedler, W., K. M. Torkar, A. Pedersen, R. Schmidt, H. Arends, F.G. Rüdenauer, M. Fehringer and Y.I. Galperin, Spacecraft control experiment RON, RSA-IKI-CNES, "Interball Mission and Payload", 1995.

Singh, N., B. I. Vashi, and L. C. Leung, A three-dimensional numerical model of body-plasma interaction, SIMPO Newsl., 3 (N9), 58-67, 1994.

Schmidt, R., H. Arends, A. Pedersen, M. Fehringer, F. Rüdenauer, W. Steiger, B. T. Narheim, R. Svenes, K. Kvernsveen, K. Tsuruda, H. Hayakawa, M. Nakamura, W. Riedler, and K. Torkar, A novel medium-energy ion emitter for active spacecraft potential control, Rev. Sci. Inst., 64 (8), 2293-2297, 1993.

Schmidt, R., H. Arends, A. Pedersen, F. Rüdenauer, M. Fehringer, B. T. Narheim, R. Svenes, K. Kvernsveen, K. Tsuruda, T. Mukai, H. Hayakawa, and M. Nakamura, Results from active spacecraft potential control on the Geotail spacecraft, J. Geophys. Res., 100 (A9), 17253-17259, 1995.

Whipple, E. C. Jr., The equilibrium electric potential of a body in the upper atmosphere and in interplanetary space, NASA Rep. X-615-65-296, Goddard Space Flight Center, Greenbelt, Md., 1965.

Zinin, L. V., Y. I. Galperin, V. A. Gladyshev, S. A. Grigoriev, L. Girard, and T. M. Muliarchik, Modelling of the anisotropic thermal plasma measurements of the energy-mass-angle ion spectrometers onboard a charged satellite, Cosmic Res., 33, 511-518, 1995. 